\title{
INDUCCION PREOPERATORIA PARA PACIENTES Y FAMILIARES EN CASO DE CIRUGIA MAYOR Y SU RELACION CON LA ANSIEDAD ${ }^{1}$
}

\author{
Rosario Vargas Mora ${ }^{2}$ \\ Cristina Herrera Sibaja ${ }^{3}$
}

Institución: Hospital Nacional de Niños Dr. Carlos Sáenz Herrera

\section{COMO CITAR}

Vargas, R., Herrera, C (2012).Inducción preoperatoria para pacientes y familiares en caso de cirugía mayor y su relación con la ansiedad.[En línea].Rev. Enfermería Actual en Costa Rica, 22, 1-11 [citado (fecha)]. Disponible World Wide Web: <http://www.revenf.ucr.ac.cr/inducción.pdf> ISSN 1409-4568

\section{RESUMEN}

A medida que avanza el conocimiento científico, los procedimientos quirúrgicos que se realizaban con pacientes hospitalizados, se llevan a cabo, ahora, de forma ambulatoria; sin embargo, es necesario preparar a los pacientes, pues ello beneficia el proceso de recuperación. El aumento de la población, los elevados costos por los servicios de salud y los avances tecnológicos, posibilitan la elección de los servicios de Cirugía Ambulatoria como una alternativa segura, rápida y eficiente para atender los procedimientos quirúrgicos de bajo riesgo, los cuales ofrecen ventajas para el paciente, familia e institución. Para este estudio se aplicó la metodología para el desarrollo de la práctica clínica basada en la evidencia. En cuanto a la necesidad de la investigación, se planteó la siguiente pregunta clínica: En los pacientes de edades entre siete a 14 años ¿La información preoperatoria acerca de su cirugía mayor ambulatoria comparada con los que no reciben información disminuye la ansiedad y presentan menos complicaciones postoperatorias?. El tipo de pregunta correspondió a una pregunta de diagnóstico. Para responder a ella, se realizó una búsqueda, revisión y análisis de la literatura científica a la que se accesó mediante el uso de buscadores como GOOGLE, administrador de artículos PUBMED, Excelencia Académica, EBSCOhost; de dichas fuentes se recuperaron 50 documentos de los cuales se encontró dos estudios de buena calidad que responden a la pregunta planteada. La educación preoperatoria intensa a familiares y al paciente pediátrico disminuye el nivel de ansiedad que generan las intervenciones quirúrgicas. El desarrollo de programas apropiados prequirúrgicos ayuda a aliviar la ansiedad de los niños y padres durante la experiencia quirúrgica.

Palabras clave: cirugía-mayor-ambulatoria, cursos, educación-preoperatoria, Enfermería, pediatría

\footnotetext{
${ }^{1}$ Fecha de recepción: 30 de noviembre 2011

${ }^{2}$ Enfermera, Hospital Nacional de Niños. Correo Electrónico: RVargasM@hnn.sa.cr

${ }^{3}$ Enfermera, Hospital Nacional de Niños. Correo Electrónico: cristina15cr@hotmail.com
}

Fecha de aceptación: 30 de enero 2012 


\title{
Revista Electrónica Enfermería Actual en costa Rica
}

\section{PREOPERATIVE INDUCTION FOR PATIENTS AND FAMILIES IN CASE OF MAJOR SURGERY AND ITS RELATIONSHIP WITH ANXIETY ${ }^{1}$}

\author{
Rosario Vargas Mora ${ }^{2}$ \\ Cristina Herrera Sibaja ${ }^{3}$
}

Institución: National Children’s Hospital Dr. Carlos Sáenz Herrera

\section{CITED}

\begin{abstract}
As scientific knowledge advances, surgical procedures were performed with hospitalized patients, currently being carried out on an outpatient basis, where the patient and family preparation is critical to patient recovery. The increasing population, high costs for health services and technological advances make possible the election of Ambulatory Surgery services as a safe, quick and efficient to meet the low-risk surgical procedures, offering advantages for the patient, family and institution. This study applied the methodology for the development of clinical practice based on evidence. Because the need was expressed in the following clinical question: In patients aged 7 to 14 years Does preoperative information about your outpatient surgery compared with those not receiving information decreases anxiety and have fewer postoperative complications? The type of question corresponded to a question of diagnosis. To answer it, we conducted a search, review and analysis of scientific literature. Which were used by different databases such as GOOGLE search engine, articles manager PUBMED, Academic Excellence, EBSCO Host. We retrieved 50 documents of which are found two good quality studies that address this question. The intensive preoperative education to family and pediatric patients decreased the level of anxiety associated with surgery. The development of appropriate pre-surgical help relieve anxiety in children and parents during the surgical experience.
\end{abstract}

Keywords: surgery-more-ambulatory, courses, education, preoperative, Nursing, pediatrics

\footnotetext{
${ }^{1}$ Date of reception: November 30, 2011

${ }^{2}$ Enfermera, Hospital Nacional de Niños. E- mail: RVargasM@hnn.sa.cr

${ }^{3}$ Enfermera, Hospital Nacional de Niños. E- mail: cristina15cr@hotmail.com
}

Date of acceptance: January 30, 2012 


\section{INTRODUCCIÓN}

A medida que avanza el conocimiento científico, los procedimientos quirúrgicos que se realizaban con pacientes hospitalizados, se llevan a cabo ahora, de forma ambulatoria; sin embargo, es necesario preparar a los pacientes, pues ello beneficia el proceso de recuperación.

El aumento de la población, los elevados costos por los servicios de salud y los avances tecnológicos posibilitan la elección de los servicios de Cirugía Ambulatoria como una alternativa segura, rápida y eficiente para atender los procedimientos quirúrgicos de bajo riesgo, los cuales ofrecen ventajas para el paciente, familia e institución; no obstante, tal opción aumenta la demanda de servicios ofrecidos por los profesionales en Enfermería, quienes, como parte de un equipo de salud, se interesan en brindar una atención de calidad, segura, que disminuya los factores de riesgo o complicaciones que se puedan presentar posterior a una cirugía.

Según la literatura revisada, es evidente que tanto niños (as) como padres no se sienten ni emocionalmente ni educativamente preparados para enfrentarse a la cirugía ambulatoria, de allí la trascendencia de desarrollar programas de inducción a los procesos quirúrgicos considerados una estrategia que ayuda a aliviar la ansiedad que genera esta experiencia.(Frisch, Johnson, Simmons y Weatherford, 2010)

El Hospital Nacional de Niños, Dr. Carlos Sáenz Herrera (HNN), cuenta con un programa de Cirugía Mayor Ambulatoria (CMA) para responder a la creciente demanda de pacientes quirúrgicos (Archivos del HNN); aunque en 1982 nació la idea de implementar Cirugía Mayor Ambulatoria, no fue hasta 1995 que la Dra. Rocío Hernández presenta a las autoridades competentes de la institución el "Proyecto para la Creación de un Centro de Atención de Cirugía Mayor Ambulatoria (CMA)”, el cual inició con algún tipo de cirugías programadas. Sin embargo, no contó con documentación científica que avalara las acciones propuestas en el programa.

Aunado a lo anterior, en 1996, las enfermeras María de los Ángeles Ruiz y Mercedes Castrillo diseñaron como proyecto de graduación en Enfermería el "Programa de Atención Integral de Enfermería al niño (a) de Cirugía Mayor Ambulatoria del Hospital Nacional de Niños" (Castrillo, 1996), un proyecto de graduación que permitió proyectar a la enfermera (o) profesional en la intervención, preparación y educación preoperatoria al paciente y familia aplicando el proceso científico de Atención de Enfermería, conocido como PAE. A pesar de tan innegable esfuerzo, no podía constatarse una verdadera articulación entre teórica científica y la práctica que se estaba realizando.

Actualmente, en este programa se atienden pacientes que precisan servicios de Otorrinolaringología, Oftalmología, Odontología, Oncología, Cirugía general, Ortopedia y Urología.

En 1999, a partir del mes de agosto, el Programa CMA es dirigido por un médico coordinador, una enfermera profesional y una auxiliar de enfermería.

Algunas de las acciones gerenciales del profesional de Enfermería se citan a continuación:

- Preparar, orientar y educar a los pacientes, padres o encargados.

- Brinda atención integral e individualizada, enfatizando en la educación de los familiares del niño (a), acerca de los cuidados pre y postoperatorios. 
- La preparación física y emocional de los niños (as) que van a ser intervenidos está bajo el cuidado del equipo de profesionales que integran el programa.

- Parte de las estrategias para la realización del programa de cirugía ambulatoria son las siguientes:

○ Curso "Inducción preoperatoria." Una vez por semana.

- Entrega de folletos educativos con información y dibujos de acuerdo con el tipo de cirugía por realizar.

- Proyección de un video (audiovisual) con toda la información concerniente al Programa CMA.

A raíz de lo expuesto, surge la inquietud de investigar si este tipo de intervención que se brinda al paciente pediátrico y su familia disminuye la ansiedad que genera la cirugía y, por lo tanto, disminuye las complicaciones posoperatorias.

Mediante la aplicación del método de Enfermería Basada en la Evidencia se incorporan elementos como el propio juicio profesional, las experiencias, las preferencias y valores de los pacientes aunado a la búsqueda de la mejor evidencia científica disponible, todo con la finalidad de poder brindar mejores cuidados enfermeros.

Actualmente, de acuerdo con Gené y Contel (2001) mencionado por Díaz et al. (2007), la Enfermería,

... ya no aspira a garantizar cuidados básicos para toda la población, sino la prestación universal de servicios de alta calidad, definidos por criterios de efectividad y coste efectividad probados. Se potencia así elementos de racionalización coherente con los valores y preferencia del paciente para responder a una demanda creciente de servicios en un contexto de recursos limitados.(s.p.)

La enfermería basada en la evidencia (EBE) brinda suficientes herramientas a los profesionales para que su práctica sea segura, confiable basada en estudios científicos y no en una práctica desarrollada tradicionalmente que en muchas ocasiones no se sabe de donde surge.

Las investigaciones han evidenciado que muchas prácticas sanitarias que se realizan no tienen justificación o razón para continuar ejecutándolas, incluso algunas de ellas son riesgosas para los pacientes. Morales (2007) nombra algunas, como el uso de enema rectal en el parto, el cual tanto Gordon (1981) como Gordon (1984) corroboraron que no favorece a la parturienta; no obstante, en algunos hospitales se sigue realizando. Otro ejemplo es el lavado gástrico en recién nacidos sanos tras parto eutócico sin presencia de meconio. Cuello-García et al. (2005) demuestran que este procedimiento está contraindicado y debe eliminarse como práctica rutinaria. Sin embargo, Díaz et al (2007) menciona que el 70\% de los hospitales en su país sigue manteniendo esa práctica.

Young (2003) insiste en que el profesional de Enfermería debe reflexionar sobre su trabajo y confiar en este para poder preguntarse acerca de su propia práctica y realizarla de la manera más segura. Siguiendo este pensamiento es que las investigadoras nos preguntamos acerca de nuestra propia práctica y de las acciones que se ejecutan en la implementación del Programa de Cirugía Mayor Ambulatoria del HNN.

El objetivo de realizar este trabajo es conseguir suficiente evidencia científica que permita fundamentar la creación del programa y realizar mejoras a este a través del fortalecimiento de las estrategias que actualmente se desarrollan para poder incorporar otras nuevas. 


\section{MATERIAL Y MÉTODOS}

Para este estudio se aplicó la metodología para el desarrollo de la práctica clínica basada en la evidencia que consta de las siguientes etapas:

- Formulación de una pregunta de primera línea en el formato P.I.C.O.

- Búsqueda de información científica disponible.

- Análisis crítico de la información.

- Implementación de los resultados encontrados en el quehacer diario del profesional.

- Evaluación de la implementación.

La pregunta formulada fue creada según el formato P.I.C.O. que se desglosa de la siguiente manera:

- Tipo de paciente o la patología de la que surge la pregunta $(\mathrm{P})$.

- La intervención que queremos analizar (I).

- La comparación con otra intervención (si procede) (C).

- Los resultados clínicos esperados $(\mathrm{O})$.

Por cuanto la necesidad se plasmó en la siguiente pregunta clínica:

Para los pacientes de edades entre siete a 14 años se planteó la siguiente interrogante. ¿Recibir información preoperatoria acerca de su cirugía mayor ambulatoria disminuye la ansiedad y presentan menos complicaciones postoperatorias en contraste con quienes no reciben información?

El tipo de pregunta correspondió a una pregunta de diagnóstico. Para responder a ella, se realizó una búsqueda, revisión y análisis de la literatura científica para la que se utilizaron diferentes bases de datos como por ejemplo buscador GOOGLE, administrador de artículos PUBMED, Excelencia Académica, EBSCOhost.

De los artículos encontrados (50), se seleccionaron por el contenido y calidad científica los dos artículos siguientes:

1. Frisch, A. Johnson, A. Simmons, S. Weatherford, C.(2010) Nurse Practitioner Role in Preparing Families for Pediatric Outpatient Surgery.

Traducido: Papel Educativo de la Enfermera Profesional para con los Pacientes de Cirugía Ambulatoria Pediátrica.

A este artículo se le aplicó la plantilla proporcionada por CASPe para evaluar la calidad.

2. Joanna Briggs Institute. (2000). Retención de la información preoperatoria recibida por los pacientes. Best Practice: Evidence based information Sheets for Health Professionals. 
Traducido por: Centro colaborador Español del Instituto Joanna Briggs para los cuidados de salud Basados en la Evidencia.

Para su valoración se le aplicó el programa FCL 2.0.

\section{RESULTADOS}

Respecto del primer estudio titulado "Papel Educativo de la Enfermera Profesional para con los Pacientes de Cirugía Ambulatoria Pediátrica", al cual se le aplicó la plantilla CASPe se obtuvo el siguiente resultado:

El estudio examinó los efectos de un programa individualizado de educación en los niveles de ansiedad y satisfacción de los padres que estuvieron activamente involucrados en la inducción del hospital y fase de recuperación de la cirugía de sus hijos.

Midió el nivel de ansiedad del paciente y familiares ante una cirugía ambulatoria. La población de estudio estuvo compuesta por 80 niños entre la edad de cinco a 11 años que tuvieron cirugía ambulatoria por la Especialidad de Otorrinolaringología, a quienes se les aplicó la técnica del “dibujo infantil”, para medir el nivel de ansiedad de los niños antes y después de la cirugía. La intervención fue aplicada a dos grupos de niños; el primer grupo constó de 80 niños con intervención educativa (se realizaron recorridos por el área quirúrgica, manipulación de equipo médico). El segundo grupo estuvo conformado por 62 niños de las mismas edades quienes sólo recibieron información general.

Ambos grupos expresaron su nivel de ansiedad a través de los dibujos. La investigación se complementó con estudios realizados en los años 2002, 2004, 2005. Por otro lado, incluyeron otros trabajos importantes y relevantes como fue el de Smith y Callery (2005) quienes realizaron un estudio cualitativo para evaluar las preocupaciones de los niños antes de la cirugía a través de otro instrumento de dibujo infantil. El ensayo clínico aleatorio de Felder- Puig et al., (2003), consistió en desarrollar un libro de colorear titulado "Conejo Maurice", suministrado a aquellos que estaban por experimentar una cirugía ambulatoria. Otro estudio evidencia los niveles de ansiedad y satisfacción de los padres previo a la cirugía de sus hijos; este estudio piloto es de Spencer y Franck (2005), autores que, investigan el momento en el que presentan la información preoperatoria.

Los autores de esta investigación, de acuerdo con el criterio de las investigadoras, han hecho suficiente esfuerzo para valorar la calidad de los trabajos científicos incluidos. Además, los autores han considerado otros estudios relevantes en los que, previo a una cirugía, aplicaron instrumentos a los niños y a sus padres tales como: dibujos, escalas, cuestionarios, material que permitió medir los diferentes niveles de ansiedad en los niños y en sus padres.

Los resultados de todos los estudios consultados están claramente definidos y hay relación entre ellos. Para validar los resultados los autores se apoyaron en otros estudios que también buscaban medir los niveles de ansiedad de los padres antes de la cirugía ambulatoria de sus hijos (Moerman, FritsVanDam, Muller y Oosting, 1996). Dicho estudio reveló la importancia de los programas de preparación previa a la cirugía ambulatoria de los niños, ya que fueron creados para disminuir la ansiedad y aumentar los conocimientos y habilidades para enfrentar la situación, de lo que se obtuvo una disminución de la ansiedad tanto en los niños como en sus padres. 
Los resultados evidenciaron además un aumento de ansiedad y miedo de los niños y sus padres cuando no han recibido educación preoperatoria.

De acuerdo con nuestro criterio, los autores citaron varios estudios de temas similares que evidenciaban resultados muy parecidos a los de la investigación, por lo tanto, se considera que los estudios citados tienen calidad para apoyar la investigación.

Los pacientes pediátricos que serán sometidos a cirugía ambulatoria se muestran menos ansiosos antes y después de la cirugía cuando reciben intervención educativa preoperatoria, lo mismo sucede con sus padres quienes se muestran muy satisfechos por la educación y el trato recibido.

El estudio demostró que los niños del primer grupo que recibieron intervención educativa preoperatoria, tales como información verbal, recorrido por el hospital y la terapia de juego de rol, mostraron menos ansiedad antes y después de la cirugía, a diferencia del segundo grupo de niños que no recibieron intervención educativa preoperatorio y que sólo recibieron información general: este grupo de niños presentó un aumento de la ansiedad.

Los resultados del estudio citado pueden aplicarse bien a nuestro medio porque los pacientes del Programa Cirugía Mayor Ambulatoria manifiestan ansiedad, temor y miedo, sobre todo los niños mayores de dos años, quienes tienen conciencia del dolor y la separación de sus padres al momento de entrar al quirófano. Igualmente, los padres expresan ansiedad, temor y miedo más si es la primera vez que su niño va a ser intervenido, de allí que la preparación previa a la cirugía sea de gran importancia.

Los investigadores han considerado todos los resultados posibles relacionados con el paciente, familia y medio.

Finalmente, en lo referente al costo/ beneficio, vale la pena la implementación de un Programa de Cirugía Mayor Ambulatoria porque, de acuerdo con los resultados del artículo investigado, hay una balanza positiva en la implementación de un programa de cirugía ambulatoria.

En cuanto al segundo estudio, Retención de la información preoperatoria recibida por los pacientes, la tabla 1 muestra los resultados obtenidos:

Tabla 1

Resultado general del análisis crítico del artículo "Retención de la información preoperatoria recibida por los pacientes" RESULTADOS CONCLUSIONES COMENTARIOS 


\begin{tabular}{|c|c|c|c|c|c|c|c|}
\hline & & & $\begin{array}{l}\text { DIAGNÓSTICA } \\
\text { PRUEBA DE } \\
\text { COMPARACIÓN }\end{array}$ & & & & LA EVIDENCIA \\
\hline $\begin{array}{l}\text { Cita } \\
\text { abreviada: } \\
\text { Hodkinson, } \\
2000 .\end{array}$ & 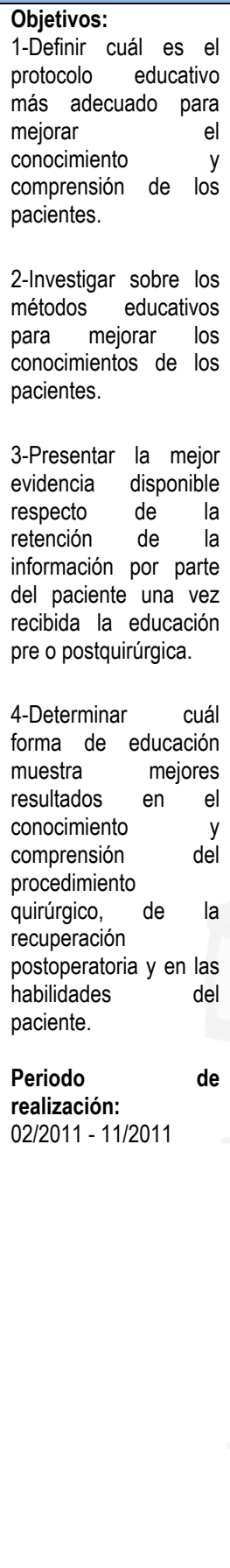 & $\begin{array}{l}\text { Número de } \\
\text { participantes: } \\
\text { Pacientes } \\
\text { femeninas que } \\
\text { se van a } \\
\text { someter a un } \\
\text { procedimiento } \\
\text { quirúrgico dos } \\
\text { Características } \\
\text { de en de } \\
\text { participantes: } \\
\text { Femeninas en } \\
\text { edad reproducción }\end{array}$ & 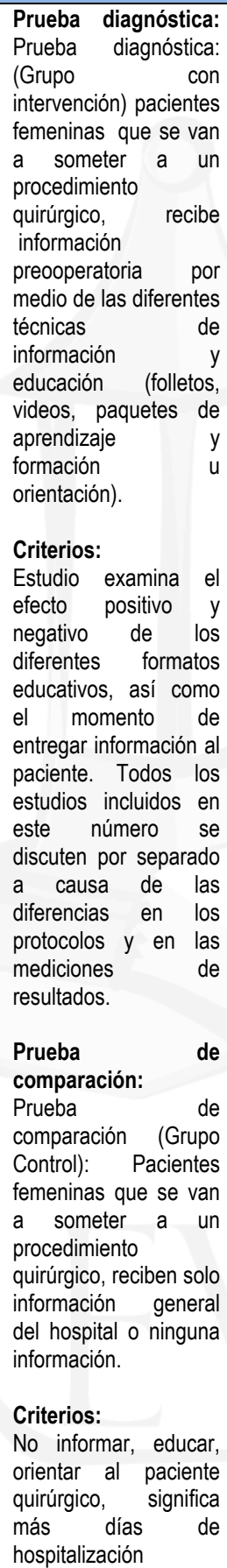 & 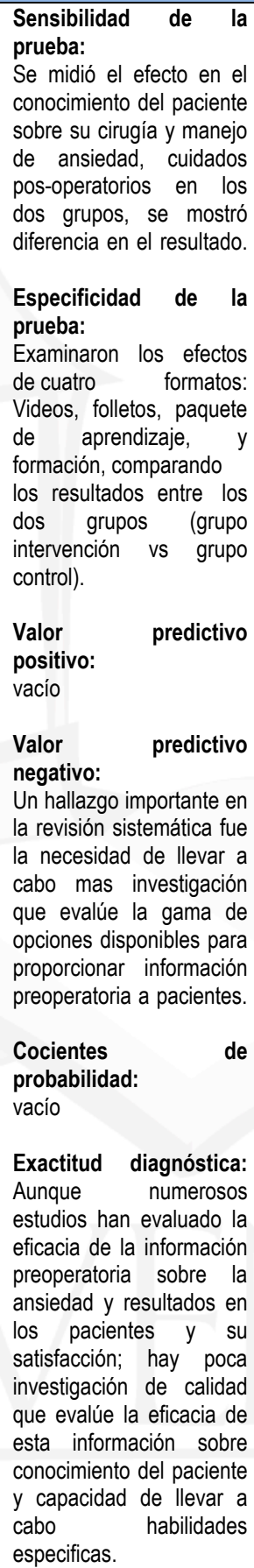 & 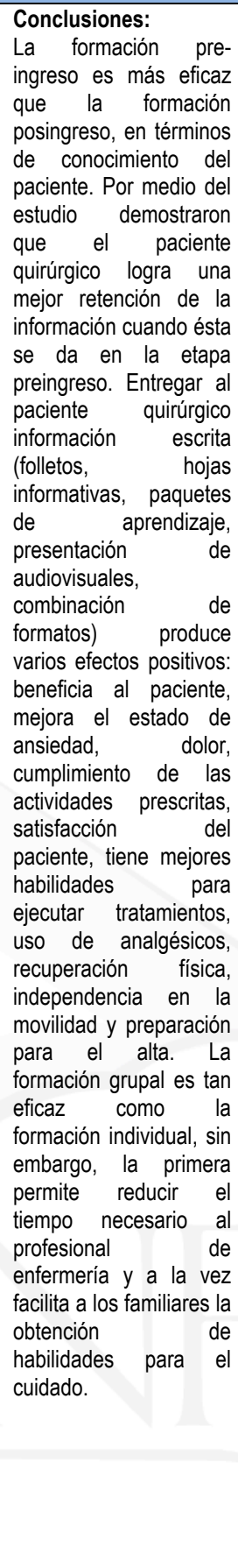 & 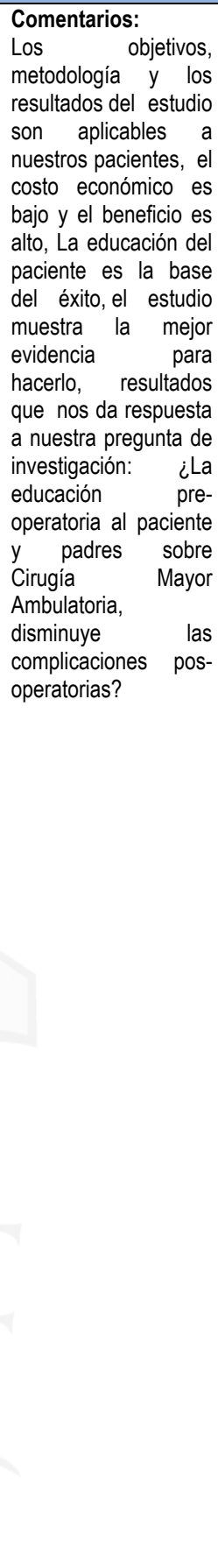 & $\begin{array}{l}\text { Calidad de la } \\
\text { evidencia: } \\
\text { Alta }\end{array}$ \\
\hline
\end{tabular}


Los resultados de ambos estudios brindan respuesta a la pregunta PICO planteada, demuestran validez y presentan resultados que apoyan la intervención educativa justo a tiempo a los pacientes pediátricos que serán sometidos a una cirugía y a sus familiares.

Los pacientes sometidos a intervención quirúrgica y sus familiares manifiestan diferentes niveles de ansiedad, ya que desde que reciben la noticia de que necesitan ser intervenidos quirúrgicamente, surge una vasta cantidad de dudas e inquietudes. Además la propia separación momentánea de los familiares durante la cirugía, Brewer et al., (2006); Shirley et al., (1998); Smith et al., (2005), el desconocimiento del lugar en donde serán intervenidos, las personas ajenas a los pacientes que lo atenderán, son factores que influyen negativamente ante la recuperación postoperatoria.

Por lo tanto, las estrategias de intervención que se han venido desarrollando en el Programa de Cirugía Mayor Ambulatoria deben seguirse ejecutando. Unas de las intervenciones que se realizan es la citación de los padres al programa una semana antes para recibir una charla de inducción; al respecto, Brewer et al., (2006) y Hatava et al., (2000) mencionados por Joanna Briggs Institute (2000) evidencian que una educación preoperatoria intensa, la cual debe incluir el juego del equipo de salud tratante y el recorrido por el hospital, disminuyen los miedos y la ansiedad experimentada por los niños y niñas que serán sometidas a intervención quirúrgica. En el programa CMA, la charla de inducción es preparada por el equipo médico y de enfermería; en ella se brinda información general a los familiares sobre el procedimiento quirúrgico que se le realizará a su hijo (a) e incluye la preparación preoperatoria que requiere, la hora de ingreso al hospital, el tiempo aproximado de permanencia y las posibles complicaciones. Se da un espacio para que los padres expresen sus dudas e inquietudes.

Con los niños se interviene cuando ya han ingresado a la unidad: si la edad lo permite se conversa con él, se le explica lo que le van a realizar, se le ofrecen libros para colorear, se agenda un recorrido por el área pre quirúrgica con el personal que intervendrá en el proceso, entre otras cosas. Sin embargo, se reconoce que debe mejorarse este tipo de intervención, utilizando videos alusivos a su cirugía, implementando ejercicios de relajación y psicoeducativos, juegos de mesa y otras actividades que le ayudarán a disminuir su ansiedad, argumento apoyado por Justus et al., (2006); Smith y Callery (2005) mencionados por Joanna Briggs Institute (2000). Como lo señala la evidencia encontrada, todo esfuerzo que se realice con los niños y padres previos a un acto quirúrgico traerá resultados muy positivos en la recuperación.

El educar y orientar genera una inversión de tiempo y dinero, así como un compromiso por parte del trabajador; sin embargo, no se compara con el beneficio para el paciente, la familia y la misma institución de salud. Los padres que reciben más educación e información presentarán niveles de tensión más bajos y estarán más tranquilos y seguros del tratamiento que recibe su hijo.

El grado de satisfacción del cliente es también un parámetro que las instituciones deben tener en cuenta. Los padres que han recibido una adecuada educación y orientación se muestran muy satisfechos al respecto.

La acción principal de la prevención es educar, por lo tanto, es una prioridad que debe tenerse en cuenta al brindar atención de calidad a los clientes. 
Como bien lo apunta la evidencia encontrada, es recomendable continuar con este tipo de prácticas e intensificar la información preoperatoria con la finalidad de que los pacientes logren una mejor recuperación y pronto alivio de su padecimiento.

\section{CONCLUSIÓN}

Existe evidencia científica que la implementación de programas apropiados prequirúrgicos que incluyen diversas estrategias como la educación, la preparación de pacientes para el procedimiento quirúrgico, el cuidado posterior, el seguimiento, la detección de alteración del estado de salud del niño (a) en su hogar, entre otras actividades, ayudan a aliviar la ansiedad de los niños y familia durante la experiencia quirúrgica.

\section{REFERENCIAS}

Brewer, S., Gleditsch, S., Syblik, D., Tietjens, M., Vacik, H. (2006). Pediatric anxiety: Child life intervention in day surgery. Journal of Pediatric Nursing, 21(1), 13- 22.

Castrillo, M. (1996). Programa de Atención Integral de Enfermería al niño (a) de Cirugía Mayor Ambulatoria del Hospital Nacional de Niños. San José, Costa Rica. S. e.

Cuello-García, C., et al. (2005). Lavado gástrico en Recién Nacidos Sanos: un Ensayo Clínico Aleatorio. Anales de Pediatría, 63 (6 ), 509-513.

Díaz, V., Álvaro, M., Campo, E., Saavedra, F. y Cabrera, M. (2007). El paradigma de la enfermería basada en la evidencia (EBE) en la enfermería clínica hospitalaria. NURE investigation, (28), 18.

Felder-Puig R, et al. (2003). Using a children's book to prepare children and parents for elective ENT surgery: results of a randomized clinical trial. International Journal of Pediatric Otorhinolaryngology, 67(1), 35-41.

Frisch, A., Johnson, A., Simmons, S., Weatherford, C.(2010). Nurse Practitioner Role in Preparing Families for Pediatric Outpatient Surgery. Pediatric Nursing. 36 (1), 41-47.

Herrera, H. (1982). Programa de Cirugía Mayor Ambulatoria. Hospital Nacional de Niños Dr. Carlos Sáenz Herrera, Cirugía, San José, Costa Rica. S. e.

Joanna Briggs Institute (2000). Knowledge Retention from pre-operative patient information. The Joanna Briggs institute for evidences based Nursing and Midwifery, 4(6), 1-6.

Moerman, N., Frits, S., Van Dam, F., Muller, M., Oosting, H. (1996). The Amsterdam preoperative anxiety and information scale (APAIS). Anesthesia Analgesia, 82, 445-451.

Morales, J. (2005) Efectividad y Evidencia: ni contigo, ni sin tí. Evidentia, 2(4). Recuperado de http://www.index-f.com/evidentia/n4/88articulo.php

Shirley, P., Thompson, N., Kenward, M. y Johnston, G. (1998). Parental anxiety before elective surgery in children. Anesthesia, 53(10), 956-959. 


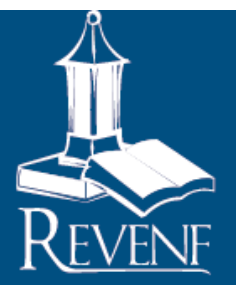

Revista Electrónica Enfermería Actual en costa Rica

Smith, L. y Callery, P. (2005). Children's accounts of their preoperative information needs. Journal of Clinical Nursing, 14(2), 230-238.

Spencer, C y Franck, L. (2005). Dar a los padres información escrita acerca de la anestesia de los niños: ¿Está el establecimiento y la sincronización importante. Anestesia Pediátrica, 15 (7), 547-553.

Young, K (2003). Where's the evidence? American Journal Nursing. 103 (10):11. 